\title{
Map-based Long Term Motion Prediction for Vehicles in Traffic Environments
}

\author{
Dominik Petrich $^{1}$, Thao Dang ${ }^{1}$, Dietmar Kasper ${ }^{1}$, Gabi Breuel ${ }^{1}$ and Christoph Stiller ${ }^{2}$
}

\begin{abstract}
Depending on driver intention and current motion state of vehicle, an infinite set of possible future trajectories exists. In this paper we present a stochastic filter which is able to select a representative set of reasonable trajectories from this solution set using additional information from a digital map. This is achieved by representing the map's traffic lanes by their corresponding centerlines. Each detected vehicle is projected on these centerlines, where the necessary information is modeled as a multivariate random variable. With this stochastic model of traffic lanes and the stochastic vector of vehicle detection we define a new stochastic residual vector, which is used both in the lane assignment and during the generation of the motion predictions. For each traffic lane, which is assigned as relevant for a detected vehicle, we generate a new motion hypothesis by using an Extended Kalman Filter. To assess the plausibility of each motion hypothesis we employ an adaptive multivariate MCUSUM algorithm. The implemented stochastic filter is tested by evaluating real data from the Ko-FAS Research Initiative. While traditional motion prediction based on previous and current motion states of a vehicle only provide plausible prediction for a short term time horizon, we show that our approach achieves a reasonable motion hypothesis for long prediction intervals especially in complex scenarios as road intersections.
\end{abstract}

\section{INTRODUCTION}

Modern Advanced Driver Assistance Systems (ADAS) have to cope with highly complex traffic scenarios, like urban intersections with many possible lanes. To handle such situations, ADAS need a comprehensive situation analysis which consists of three main objectives [1]: the representation and interpretation of the current traffic situation, the prediction of the dynamic evolvement of the scene, and criticality assessment. Especially the evolution of the current situation in complex traffic scenarios like multi-lane intersections are challenging.

Because vehicles operate on a constrained environment of road networks, future trajectories are influenced by the layout of the road. Assuming a compliant behavior of traffic participants, knowledge of the road's layout permits representative long term motion predictions.

Several works from recent years which are dealing with long term motion prediction utilize information about the previous states of a vehicle to predict its future trajectory [2]. Otter works consider the boundary conditions of the road layout to infer the driver's intent [3].

\footnotetext{
${ }^{1}$ The authors are with the Daimler AG, RD/driving-automation, Boeblingen, Germany \{dominik.petrich, thao.dang, dietmar.kasper, gabi.breuel\}edaimler.com

${ }^{2}$ The author is with the Institute of Measurement and Control Systems, Karlsruher Institute of Technology, Germany stiller@kit.edu
}

However, most existing approaches use the information of the road on which the vehicle is traveling to improve the state estimation. The main idea of these proposals is to use the map information as state constraints. The applied procedures can be divided into several groups [4]. The first one is to incorporate road information directly into the state estimation process using variable structure interacting multiple models (VS-IMM) methods [5] or Multi-Hypothesis Tracking (MHT) [6]. The second group projects unconstrained state estimates of a Kalman filter step onto the constrained surface [7]. Another method is to project a dynamic system onto the state constraints and then apply the Kalman filter to the projected system [8], [9]. The approach presented in this paper treats state constraints as pseudo measurements in the spirit of [4] and [10], which forms an additional group. The advantage of this method is that the analytic model of a road segment not only weakly constrains the position but also the direction of motion. Furthermore, an estimate of centripetal acceleration can be obtained given the road curvature and the vehicle speed. This enables us to define the succeeding control input function to estimate the future trajectories for the dynamic system.

The main objective of this paper is to determine the set of reasonable future trajectories for all detected vehicles in the current traffic situation. This allows us to predict the dynamic evolvement of the scene and in turn to react to potentially hazardous situations as early as possible.

This paper is organized as follows: In Section II a descriptive model of the current situation is introduced. To combine the particular vehicle entities with additional map data the structure of the map and the definition of the traffic lane assignment is explained in Section III. Based on this, the strategy for creating specific motion hypotheses is defined in Section IV. Next in Section V, a new assessment of the motion hypothesis is discussed. Finally the presented algorithm is tested with some real data from the Ko-FAS Research Initiative [11] in Section VI and the results are summarized in Section VII.

\section{SITUATION MODEL}

A reasonable prediction how the current situation will evolve in the next few seconds requires a comprehensive knowledge of the situation at current time $t_{0}$. Due to the uncertainties of the data fusion layer and the stochastic disturbances, the initial situation $\mathcal{S}$ can be formulated as stochastic state vector model, as shown in [1]:

$$
\mathcal{S}\left(t_{0}\right)=\left\{\mathcal{X}_{i}\left(t_{0}\right) \mid i \in[1, N]\right\}
$$


where $\mathcal{X}_{i}\left(t_{0}\right), i \in[1, N]$ denotes the stochastic representation of states (pose, velocity, shape, etc.) of all $N$ objects in the scene including the ego vehicle. The random vector $\mathcal{X}_{i}$ is modeled by a normal distribution

$$
\mathcal{X}_{i} \sim \mathcal{N}\left(\mathbf{x}_{i}, \boldsymbol{\Sigma}_{i}\right)
$$

with expectation vector $\mathbf{x}_{i}$ and covariance matrix $\boldsymbol{\Sigma}_{i}$.

The evolution of this initial situation is determined by the future states of the individual vehicles. In this paper, we conduct motion prediction through a probabilistic model using the Extended Kalman Filter (EKF) [12], which allows us to incorporate knowledge of system dynamics and additional information from an external source (e.g. sensors or digital map). We use a time-discrete representation of prediction time interval

$$
T^{K}=\left\{t(k)=t_{0}+k \Delta t \mid k \in\{0,1, \ldots, K\}\right\}
$$

with $K$ discrete, equidistant time steps.

Beyond sensor data, we employ additional information extracted from a digital map assuming that an observed vehicle is traveling on a known lane.

\section{ASSIGNMENT OF LANES}

Beside the information provided from the sensor fusion layer the fundamental input data of the proposed approach is a digital map which comprises all lanes on public roads. To produce reasonable predictions of future motion states, each lane has to be associated with the detected vehicle state. In this section we present the design of the used map and an efficient method to associate a vehicle with lanes in its vicinity.

\section{A. Design of the Map}

To provide the necessary information for the motion prediction of a detected vehicle each lane of the map is represented by its corresponding centerline $\left(\iota_{i}\right)$. These centerlines are composed of successive waypoints $\left(\nu_{j}\right)$, defined in WGS 84 coordinates [13] and additional data, e.g. successor lanes and attributed traffic signs. The movement direction of a lane is given by the sequential arrangement of the waypoints. In order to simplify the computation of the motion prediction, all available data from the digital map and sensor system is transformed into the same cartesian coordinate system $\Lambda_{0}$. An example of a road segment with two lanes is shown in Fig. 1.

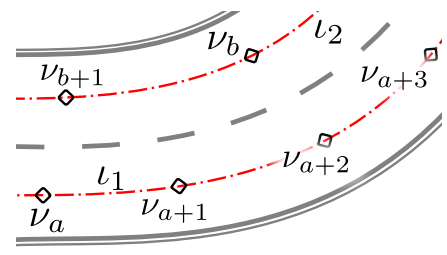

Fig. 1. Road segment with centerlines $\iota_{i}$ (red dash line) defined through consecutive waypoints $\nu$ (rotated rectangles)

Given four successive waypoints $\nu_{0,1,2,3}$ the centerline between point $\nu_{1}$ and $\nu_{2}$ can be interpolated by a cubic optimized geometric Hermite $(\mathrm{OGH})$ curve $\mathbf{Q}(u), u \in[0,1]$, [14]. Satisfying $\left(\mathbf{Q}^{\prime}=\frac{\partial}{\partial u} \mathbf{Q}\right)$

$$
\begin{aligned}
\mathbf{Q}(u=0) & =\boldsymbol{\nu}_{1}, \quad \mathbf{Q}(u=1)=\boldsymbol{\nu}_{2}, \\
\mathbf{Q}^{\prime}(u=0) & =\alpha_{1} \mathbf{T}_{1}, \quad \mathbf{Q}^{\prime}(u=1)=\alpha_{2} \mathbf{T}_{2},
\end{aligned}
$$

a cubic OGH curve is mathematically and geometrically smooth, i.e. the strain energy is minimal and the curve is cusp-, loop- and fold-free. This can be achieved by optimizing the length of the endpoint tangent vectors $\mathbf{T}_{1,2}$ with the optimized coefficients $\alpha_{1,2}$. Therefore, the geometric smoothness conditions

$$
\cos \left(\theta_{1}\right)=\frac{\mathbf{T}_{1} \mathbf{T}_{0}}{\left|\mathbf{T}_{1}\right|\left|\mathbf{T}_{0}\right|}>\frac{1}{3}, \text { and } \cos \left(\theta_{2}\right)=\frac{\mathbf{T}_{2} \mathbf{T}_{0}}{\left|\mathbf{T}_{2}\right|\left|\mathbf{T}_{0}\right|}>\frac{1}{3},
$$

have to be fulfilled with

$$
\mathbf{T}_{0}=\boldsymbol{\nu}_{2}-\boldsymbol{\nu}_{1}, \quad \mathbf{T}_{1}=\boldsymbol{\nu}_{2}-\boldsymbol{\nu}_{0}, \quad \mathbf{T}_{2}=\boldsymbol{\nu}_{3}-\boldsymbol{\nu}_{1}
$$

Furthermore, the values of the optimized coefficients $\alpha_{1,2}$ must be positive. These coefficients are defined by

$$
\begin{aligned}
\alpha_{1} & =\frac{6\left[\left(\boldsymbol{\nu}_{1}-\boldsymbol{\nu}_{2}\right) \cdot \mathbf{T}_{1}\right]\left(\mathbf{T}_{2}^{2}\right)-3\left[\left(\boldsymbol{\nu}_{2}-\boldsymbol{\nu}_{1}\right) \cdot \mathbf{T}_{1}\right]\left(\mathbf{T}_{1} \cdot \mathbf{T}_{2}\right)}{4\left(\mathbf{T}_{1}^{2}\right)\left(\mathbf{T}_{2}^{2}\right)-\left(\mathbf{T}_{1} \cdot \mathbf{T}_{2}\right)^{2}}>0 \\
\alpha_{2} & =\frac{3\left[\left(\boldsymbol{\nu}_{1}-\boldsymbol{\nu}_{2}\right) \cdot \mathbf{T}_{1}\right]\left(\mathbf{T}_{1} \cdot \mathbf{T}_{2}\right)-6\left[\left(\boldsymbol{\nu}_{1}-\boldsymbol{\nu}_{2}\right) \cdot \mathbf{T}_{1}\right]\left(\mathbf{T}_{2}^{2}\right)}{\left(\mathbf{T}_{1} \cdot \mathbf{T}_{2}\right)^{2}-4\left(\mathbf{T}_{1}^{2}\right)\left(\mathbf{T}_{2}^{2}\right)}>0 .
\end{aligned}
$$

Due to our definition of waypoints of a lane these constraints are always satisfied. In this manner, the centerline can be represented piecewise by a differentiable function of third order

$$
\begin{aligned}
\mathbf{Q}(u)= & {\left[2\left(\boldsymbol{\nu}_{1}-\boldsymbol{\nu}_{2}\right)+\alpha_{1} \mathbf{T}_{1}+\alpha_{2} \mathbf{T}_{2}\right] \cdot u^{3} } \\
& +\left[3\left(\boldsymbol{\nu}_{2}-\boldsymbol{\nu}_{1}\right)-2 \alpha_{1} \mathbf{T}_{1}-\alpha_{2} \mathbf{T}_{1}\right] \cdot u^{2} \\
& +\left[\alpha_{1} \mathbf{T}_{1}\right] \cdot u+\boldsymbol{\nu}_{1} .
\end{aligned}
$$

In the next section an efficient method is introduced to assign vehicles to individual lanes.

\section{B. Active Lane Points}

As noted before, single lanes can be formulated as piecewise mathematically and geometrically smooth functions. To associate a given vehicle with such a lane model, the geometric position $\left\{x_{i}\left(t_{0}\right), y_{i}\left(t_{0}\right)\right\}$ of the expectation vector $\mathbf{x}_{i}\left(t_{0}\right)$ is projected perpendicular to the course of the centerline, as shown in Fig. 2. In this paper the projected point on the current lane is called Active Lane Point (ALP).

Both the process of determining the position of the waypoints and the interpolation of the center line is fraught with uncertainties. To take these uncertainties into account the ALP is modeled as a multivariate stochastic variable $\mathcal{X}_{A L P}$ with a normal distribution function

$$
\mathcal{X}_{A L P} \sim \mathcal{N}\left(\mathbf{x}_{A L P}, \boldsymbol{\Sigma}_{A L P}\right)
$$

where $\mathbf{x}_{A L P}$ denotes the expectation vector at the projected position on the centerline and $\boldsymbol{\Sigma}_{A L P}$ defines the covariance matrix. As already mentioned, the map information will be used to establish a prediction of the vehicle movement along relevant lanes. For this reason, the stochastic variable in ALP includes the same state variables as the motion model contains for dynamic objects, like pose $\{x, y, \psi\}$ 


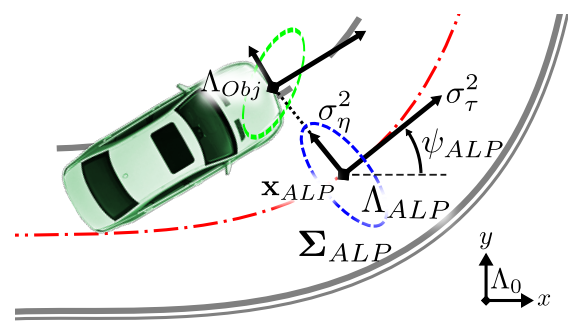

Fig. 2. Active Lane Point (ALP) on centerline with two dimensional covariance (blue)

and permitted velocity $v$. The uncertainties of this mapbased informations is stored in the covariance matrix of the probability density function (pdf), see also [4]. To use only one representation for this uncertainties, the covariance matrix is defined in the local ALP coordinate system $\Lambda_{A L P}$ and transformed into the global system $\Lambda_{0}$ with

$$
\boldsymbol{\Sigma}_{A L P}^{\Lambda_{0}}=\mathbf{T}_{\Lambda_{A L P}}^{\Lambda_{0}} \boldsymbol{\Sigma}_{A L P}^{\Lambda_{A L P}}\left(\mathbf{T}_{\Lambda_{A L P}}^{\Lambda_{0}}\right)^{T}
$$

The definition of the covariance matrix in the coordinate system of the ALP has the advantage that the variances tangential $\sigma_{\tau}^{2}$ and normal $\sigma_{\eta}^{2}$ to the centerline may be considered to be uncorrelated. The variances of the orientation $\sigma_{\psi}^{2}$ and the velocity $\sigma_{v}^{2}$ are constant in every coordinate system, so the transformation matrix becomes

$$
\mathbf{T}_{\Lambda_{A L P}}^{\Lambda_{0}}=\left(\begin{array}{cccc}
\cos \psi_{A L P} & -\sin \psi_{A L P} & 0 & 0 \\
\sin \psi_{A L P} & \cos \psi_{A L P} & 0 & 0 \\
0 & 0 & 1 & 0 \\
0 & 0 & 0 & 1
\end{array}\right)
$$

where $\psi_{A L P}$ denotes the rotation angle. This angle describes the orientation of the centerline in the ALP and can be calculated with the first deviation of the OHG function:

$$
\psi_{A L P}=\arctan \left(\frac{Q_{y}^{\prime}\left(u_{A L P}\right)}{Q_{x}^{\prime}\left(u_{A L P}\right)}\right) .
$$

Since no traffic participant drives exactly on a centerline, the deviation from detected vehicle and ALP state vector can be defined by this pdf. In this case the pdf in the ALP represents a probabilistic position model for objects on lanes within which the error of the lane model is over approximated.

\section{Traffic Lane Assignment}

A main difficulty in map-based motion prediction is the assignment of a detected vehicle to surrounding centerlines, especially on roads with several lanes in each direction or at intersections. To overcome this problem we define the stochastic residual vector $\mathcal{E}_{\text {lane }}$ between the random variables of a detected object ${ }^{1} \mathcal{X}_{O b j}$ and the ALP of a particular lane $\mathcal{X}_{A L P}$ by

$$
\mathcal{E}_{\text {lane }}=\mathcal{X}_{A L P}-\mathcal{X}_{O b j}
$$

Because of non correlating data between sensor system and ALP the covariance matrix of this normal distributed residual

\footnotetext{
${ }^{1}$ objects are detected vehicles from the sensor fusion layer
}

is given by

$$
\boldsymbol{\Sigma}_{\text {lane }}=\boldsymbol{\Sigma}_{A L P}+\boldsymbol{\Sigma}_{O b j}
$$

In probability and statistics a common test to evaluate normal distributed residuals is the $\chi^{2}$-test, which is based on the squared Mahalanobis distance:

$$
\mathcal{M}_{\text {lane }}^{2}=\mathbf{e}_{\text {lane }}^{T} \boldsymbol{\Sigma}_{\text {lane }}^{-1} \mathbf{e}_{\text {lane }},
$$

where $\mathbf{e}_{\text {lane }}$ is a realization of the random variable $\mathcal{E}_{\text {lane }}$. As a quadratic function of normally distributed random variables, the squared Mahalanobis is $\chi_{f}^{2}$-distributed. Here, the degree of freedom $f$ correlates with the number of state variables.

The quality criterion of the $\chi^{2}$-test is defined by

$$
\mathcal{L}_{\text {lane }}:=\operatorname{Pr}\left\{\chi_{f}^{2}>\mathcal{M}_{\text {lane }}^{2}\right\}=\int_{\mathcal{M}_{\text {lane }}^{2}}^{\infty} p_{\chi_{f}^{2}}(\xi) d \xi,
$$

which can be used to determine a significance level $\mathcal{L}_{\text {lane }}$ of the given realization. If this level is below an a-priori defined threshold $\left(\mathcal{L}_{\text {lane }}<\mathcal{L}_{\text {min }}\right)$, e $\mathbf{e}_{\text {lane }}$ is considered as an unlikely realization of $\mathcal{E}_{\text {lane }}$ and is discarded. In this paper a high significance level is equivalent to a plausible hypothesis that a vehicle is driving on a particular lane.

\section{MOTION HYPOTHESIS}

Throughout this paper an appropriate motion hypothesis will be generated for each lane which was classified as relevant for a detected vehicle at time step $k=t_{0}$. This means, the significance level of the hypothesis, cf. Eqn. 16, is higher than $\mathcal{L}_{\text {min }}$.

If a lane allows more than one maneuver possibility (e.g. left turn, right turn and straight), each of them is covered by a separate motion hypothesis. A motion hypothesis consists of a sequence of $K$ states of the vehicle $\mathbf{x}_{O b j}$ and the associated covariance $\boldsymbol{\Sigma}_{O b j}$. It defines the future trajectory

$$
\mathbf{X}_{O b j}=\left\{\mathcal{X}_{O b j}(k) \sim \mathcal{N}\left(\mathbf{x}_{O b j}(k), \boldsymbol{\Sigma}_{O b j}(k)\right) \mid k \in T^{K}\right\} .
$$

As already mentioned, prediction is obtained by an Extended Kalman Filter estimation process. Within the time interval $T^{K}$ the future object trajectory can be obtained from the EKF which consists of two alternating steps: The prediction step subsequently utilizes a motion model to generate object states at futur time steps, the update step incorporates lane information to refine the generated object states.

The particular steps will be explained in the following subsections. Fig. 3 shows the entire prediction process for one motion hypothesis.

\section{A. Prediction}

For vehicles, the motion model is specified as a kinematic bicycle model [15]. Therefore, the control input function is given by wheel angle $\delta_{k}$ and longitudinal acceleration $a_{k}$. To estimate these control inputs two assumptions have been made in the EKF algorithm:

1) Constant acceleration $a_{k}=a\left(t_{0}\right)$, constrained by permitted lane velocity and vehicle standstill, i.e. $v_{k} \in\left[0, v_{\max }\right]$. 


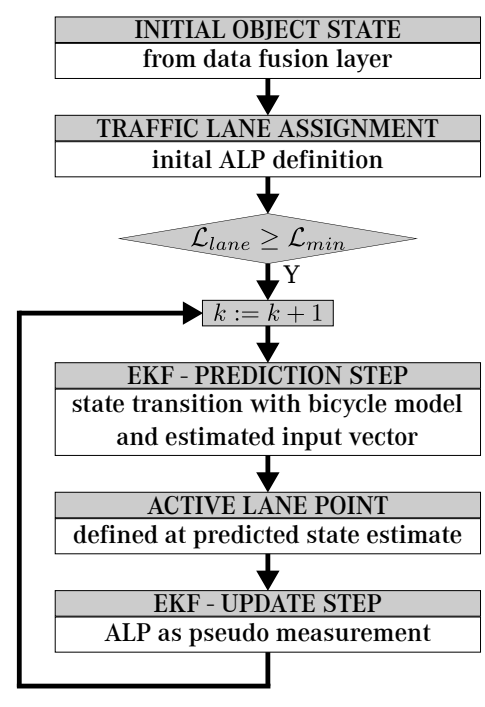

Fig. 3. motion prediction process with extended Kalman filter including the information of a lane

2) The vehicle follows curvature and orientation of the lane. Accordingly the wheel angle $\delta_{k}$ is determined due the curvature at the ALP $\left(\delta_{\text {curve }, k}\right)$ and the difference between the orientation of the object to the ALP $\left(\delta_{\Delta \psi, k}\right)$

$$
\delta_{k}=\delta_{\text {curve }, k}+\delta_{\Delta \psi, k}
$$

Taking these assumptions into account the state estimate can be expressed by removing the system noise from state transition function of the EKF:

$$
\hat{\mathbf{x}}_{k+1}^{-}=\mathbf{f}\left(\hat{\mathbf{x}}_{k}, \mathbf{u}_{k}, \mathbf{0}\right) .
$$

Here, $\mathbf{f}$ represents the non-linear bicycle model, $\hat{\mathbf{x}}_{k}$ is the current estimation of the object state and $\mathbf{u}_{k}=\left(\begin{array}{ll}\delta_{k} & a_{k}\end{array}\right)^{T}$ is the control input of the bicycle model.

The a-priori estimate covariance matrix is given by

$$
\mathbf{P}_{k+1}^{-}=\mathbf{F}_{k+1} \mathbf{P}_{k} \mathbf{F}_{k+1}^{T}+\mathbf{W}_{k+1} \mathbf{Q}_{k} \mathbf{W}_{k+1}^{T},
$$

where $\mathbf{F}$ and $\mathbf{W}$ denote the Jacobian matrices, which are evaluated at current predicted states. We assume that the process noise influences only the values of the control input, so the covariance matrix $\mathbf{Q}_{k}$ reduces to

$$
\mathbf{Q}=\left(\begin{array}{cc}
\sigma_{\delta}^{2} & 0 \\
0 & \sigma_{a}^{2}
\end{array}\right)
$$

which remains constant over the motion prediction process.

\section{B. Update}

The update step corrects the state prediction considering a new measurement. Due to the fact that we want to predict future object states for a long time interval, no additional measurement is possible. To influence the predicted object states with course information of the lane, the Active Lane Point can be used as pseudo-measurement $\mathbf{z}_{k}=\mathbf{x}_{A L P, k}$ as proposed in [4] and [10].
Since the ALP has the same attributes as the object state the measurement residual is

$$
\tilde{\mathbf{r}}_{k}=\mathbf{z}_{k}-\mathbf{H} \hat{\mathbf{x}}_{k}
$$

where the observation matrix $\mathbf{H}$ is equal to identity matrix I. As a consequence the residual covariance becomes

$$
\boldsymbol{\Sigma}_{k}=\mathbf{P}_{k}^{-}+\boldsymbol{\Sigma}_{A L P, k}
$$

and the near-optimal Kalman Gain of the EKF simplifies to

$$
\mathcal{K}_{k}=\mathbf{P}_{k}^{-} \boldsymbol{\Sigma}_{k}^{-1}
$$

With $\mathbf{z}_{k}=\mathbf{x}_{A L P, k}$ and $\mathbf{H}=\mathbf{I}$ the updated state estimate is determined by

$$
\hat{\mathbf{x}}_{k}=\hat{\mathbf{x}}_{k}^{-}+\mathcal{K}_{k}\left(\mathbf{x}_{A L P, k}-\hat{\mathbf{x}}_{k}^{-}\right),
$$

and the a-posteriori estimate covariance matrix is given by

$$
\mathbf{P}_{k}=\left(\mathbf{I}-\mathcal{K}_{k}\right) \mathbf{P}_{k}^{-}
$$

Depending on the relative values of the variances in the covariance matrices of $\mathcal{X}_{A L P}$ and $\mathcal{X}_{O b j}$ the updated state estimate is affected more or less by the pseudo measurement. If the chosen variances of the ALP in $\boldsymbol{\Sigma}_{A L P}$ is large in relation to the information of a detected object in $\boldsymbol{\Sigma}_{O b j}$, more lanes are assessed as relevant. Simultaneously the motion prediction is less influenced by the course of a lane.

\section{ASSESSMENT OF MOTION HYPOTHESES}

To assess the plausibility of a motion hypothesis we use the development over time of the stochastic residual vector $\mathcal{E}$ between object and ALP state of the corresponding centerline. In this work we employ the MCUSUM (multivariate cumulative sum) algorithm, which is a sequential analysis technique for detecting small and moderate mean shift in multidimensional process variables [16].

\section{A. Standard MCUSUM}

In the standard MCUSUM algorithm [16], the divergence vector $\mathcal{D}$ of the MCUSUM is evaluated at time step $k$ as:

$$
\mathcal{D}_{k}= \begin{cases}\mathbf{0}, & \text { if } c_{k} \leq \mathcal{B} \\ \left(1-\frac{\mathcal{B}}{c_{k}}\right)\left(\mathcal{D}_{k-1}+\mathbf{e}_{k}\right), & \text { if } c_{k}>\mathcal{B}\end{cases}
$$

where $\mathbf{e}_{k}$ is the realization of the stochastic residual vector $\mathcal{E}_{k}$ and $\mathcal{B}$ is a predetermined statistical distance. Please note that $k$ denotes measurement time steps and no longer prediction time steps. For MCUSUM we define $k>0$ and $\mathcal{D}_{0}=0$.

The distance $c_{k}$ is calculated by the current realization and the divergence vector at previous time step $k-1$

$$
c_{k}=\sqrt{\left(\mathcal{D}_{k-1}+\mathbf{e}_{k}\right)^{T} \boldsymbol{\Sigma}_{\text {lane }}^{-1}\left(\mathcal{D}_{k-1}+\mathbf{e}_{k}\right)} .
$$

In analogy to lane assignment shown in Section III, the test statistic of MCUSUM is defined as the Mahalanobis distance of the divergence vector

$$
\mathcal{M}_{\mathcal{D}, k}=\sqrt{\mathcal{D}_{k}^{T} \boldsymbol{\Sigma}^{-1} \mathcal{D}_{k}}
$$




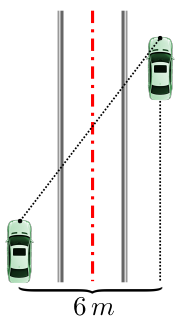

(a) Maneuver 1

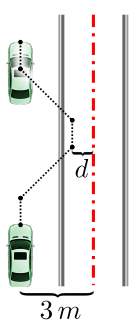

(b) Maneuver 2
Fig. 4. Two motion maneuvers to review the results of the assessment methods

where the squared value can be evaluated again by the $\chi^{2}$-test (16). Thus we get a history based significance level for each centerline motion hypothesis, which produces better results for object with large centerline offsets or for noisy signals as will be shown in Section VI.

Due to the fact, that the MCUSUM originates from statistical quality control, the algorithm recognizes efficiently if measured data do not fit to an assumed hypothesis. But if a detected object makes a lane change, the significance level of the motion hypothesis on the new lane will be too small, because the divergence vector is very high at the beginning of the maneuver. For this reason, we need an adaptive MCUSUM which considers the motion direction of an object.

\section{B. Adaptive MCUSUM}

To resolve the mentioned issues the previous divergence vector $\mathcal{D}_{k-1}$ have to be reduced when the object moves to the centerline. For this purpose, we introduce a new adaptive MCUSUM by modifying the previous divergence vector with the Mahalanobis distance of the current and previous residual vector $\mathbf{e}_{\text {lane }, k}$ (cf. Eqn. (15))

$$
\mathcal{D}_{k-1}^{* *}= \begin{cases}\left(\frac{\mathcal{M}_{\text {lane }, k}-\mathcal{B}}{\mathcal{M}_{\text {lane }, k-1}}\right) \mathcal{D}_{k-1}^{*}, & \text { if } \mathcal{M}_{\text {lane }, k}, c_{k}>\mathcal{B} \\ \mathbf{0}, & \text { else }\end{cases}
$$

The new test statistic is given by

$$
c_{k}^{*}=\sqrt{\left(\mathcal{D}_{k-1}^{* *}+\mathbf{e}_{k}\right)^{T} \boldsymbol{\Sigma}_{\text {lane }}^{-1}\left(\mathcal{D}_{k-1}^{* *}+\mathbf{e}_{k}\right)},
$$

Analogously to (27), the adapted divergence vector of the current observation is given by:

$$
\mathcal{D}_{k}^{*}= \begin{cases}\mathbf{0}, & \text { if } c_{k}^{*} \leq \mathcal{B} \\ \left(1-\frac{\mathcal{B}}{c_{k}^{*}}\right)\left(\mathcal{D}_{k-1}^{* *}+\mathbf{e}_{k}\right), & \text { if } c_{k}^{*}>\mathcal{B}\end{cases}
$$

Equal to normal MCUSUM algorithm, the assessment of motion hypothesis is based on the significance level (16), which is here determined by the squared Mahalanobis distance $\mathcal{M}_{\mathcal{D}^{*}, k}$ of the adaptive divergence vector $\mathcal{D}_{k}^{*}$. Fig. 5 represents the results of the assessment methods for two simple motion maneuver as shown in Fig. 4. For the sake of clarity we reduce the residual vector e to a scalar which represents the perpendicular distance between vehicle and centerline. In all examples the predetermined distance is set
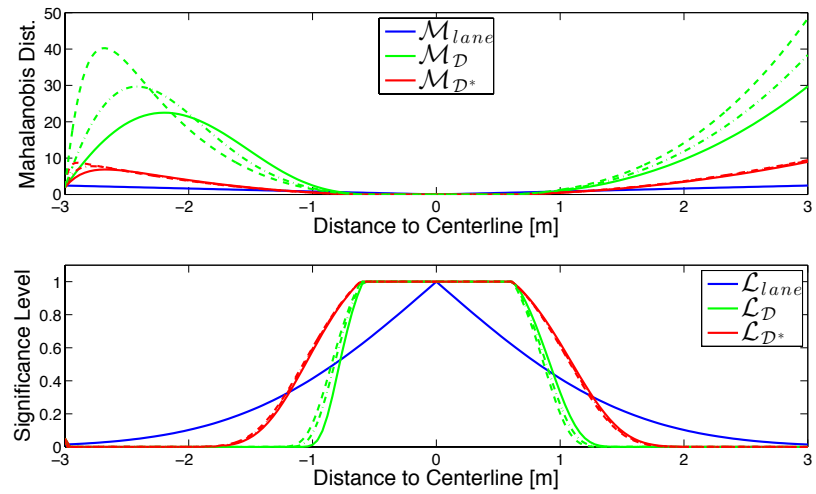

(a) Maneuver 1: vehicle moves over a distance of $4 \mathrm{~m}$ with various lateral velocities across a lane $(2 \mathrm{~m} / \mathrm{s}$ : solid, $1 \mathrm{~m} / \mathrm{s}$ : dash-dot, $0.5 \mathrm{~m} / \mathrm{s}$ : dashed). As one can see, the velocity of a vehicle has no effect on the assessment of the significance level. Furthermore, the adaptive MCUSUM $\left(\mathcal{L}_{\mathcal{D}^{*}}\right)$ produces smoother results as the significance level from the stochastic residual vector $\left(\mathcal{L}_{\text {lane }}\right)$ without generating large divergence vectors like the standard MCUSUM $\left(\mathcal{L}_{\mathcal{D}}\right)$.
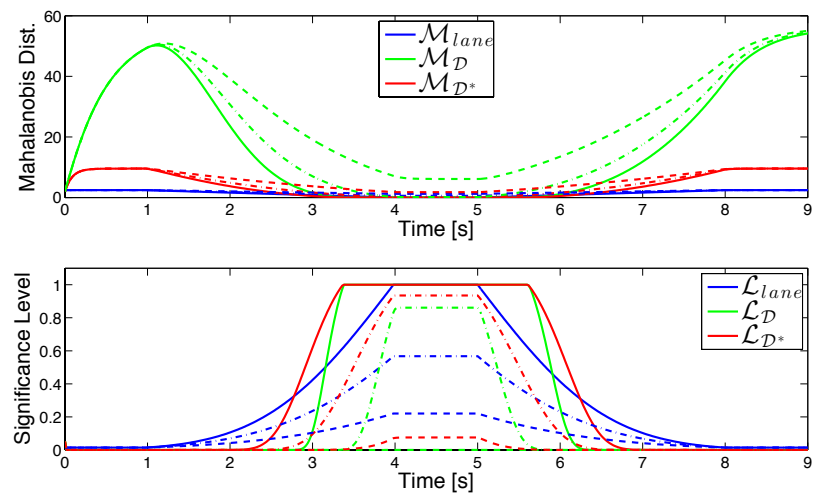

(b) Maneuver 2: vehicle moves with a constant distance of $3 \mathrm{~m}$ beside the current lane, approaches the centerline to various small distances and departs again from it. Distances $d$ in the middle of the maneuver: $d_{1}=0 m$ (solid), $d_{2}=0.7 m$ (dash-dot), $d_{3}=1.5 m$ (dashed); distance at the end is always $3 \mathrm{~m}$. In this case we can see that the adaptive MCUSUM delivers stronger decision if a traffic lane is relevant to a vehicle or not.

Fig. 5. Mahalanobis distance $(\mathcal{M})$ and significance level $(\mathcal{L})$ of normal $(\mathcal{D})$ and adaptive $\left(\mathcal{D}^{*}\right)$ MCUSUM for a traffic lane at two simple motion maneuvers

to $\mathcal{B}=0.5$ and combined variance of the stochastic residual is set to $\sigma_{\text {lane }}^{2}=1.5$ with $\sigma_{O b j}^{2}=0.5$ and $\sigma_{A L P}^{2}=1$. As shown in Fig. 5.a, both standard and adaptive MCUSUM enables a more robust decision making to decide whether a lane is relevant for the vehicle or not. Since the impact of previous divergence vectors is reduced in the adaptive MCUSUM, it responds faster and with higher significance level than the standard algorithm, as illustrated in Fig. 5.b.

\section{EXPERIMENTAL RESULTS}

This section presents some results of the long-term motion prediction algorithm evaluating real data from an intersection at Aschaffenburg, Germany, which is provided by the KoFAS Research Initiative [11]. The perception of the environment is realized by sensors in vehicles, which are complemented and supported by stationary sensor networks at the intersection. The used information on the traffic environment 


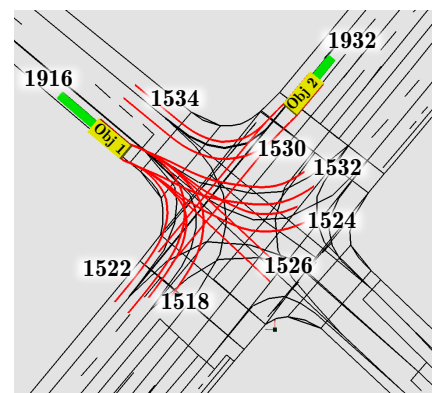

(a) $\mathbf{t}=\mathbf{2 . 8} \mathrm{s}$ : $\mathrm{Obj} 1$ is assigned to lane $1916, \mathrm{Obj} 2$ to lane 1530 and 1534
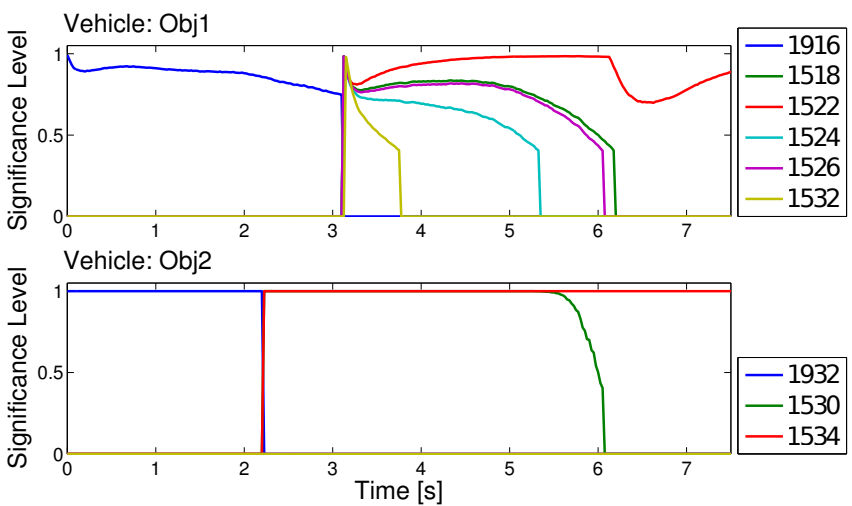

(c) History of the significance level of particular lanes assigned to both vehicles Obj1 and Obj2. The results are represented as follows: current Mahalanobis distance as dash dot line, standard and adaptive MCUSUM as dashed respectively solid line.

Fig. 6. Evaluation of the lane assignment for two detected vehicles at an intersection. Figure (a) and (b) show plausible motion prediction for the vehicles at two different times, which are determined by the red edges of the corresponding future motion path. The curve of resulting significance level over time is shown in (c)

provided from the sensor fusion layer is processed every 20 $\mathrm{ms}$ from the situation analysis layer. The digital map with the individual lanes and centerlines is stored local in a database.

For the stochastic representation of the centerline at the ALP we define the values of the uncorrelated variances to be $\sigma_{A L P, \tau}^{2}=0.3$ and $\sigma_{A L P, \eta}^{2}=1.5$ for position in ALP coordinates, $\sigma_{A L P, \psi}^{2}=0.5$ for orientation and $\sigma_{A L P, v}^{2}=0.1$ for velocity. In this way, the covariance matrix of the stochastic variable $\mathcal{X}_{A L P}$ in ALP-coordinates becomes

$$
\boldsymbol{\Sigma}_{A L P}^{\Lambda_{A L P}}=\left(\begin{array}{cccc}
0.3 & 0 & 0 & 0 \\
0 & 1.5 & 0 & 0 \\
0 & 0 & 0.5 & 0 \\
0 & 0 & 0 & 0.1
\end{array}\right)
$$

In order to enhance comparability between the particular examples, we modeled the same diagonal covariance matrix for all vehicle measurements with $\sigma_{O b j, x}^{2}\left(t_{0}\right)=0.5$ and $\sigma_{O b j, y}^{2}\left(t_{0}\right)=0.5$ for position, $\sigma_{O b j, \psi}^{2}\left(t_{0}\right)=0.1$ for orientation and $\sigma_{O b j, v}^{2}\left(t_{0}\right)=0.1$ for velocity. This leads to following covariance matrix

$$
\Sigma_{O b j}^{\Lambda_{0}}\left(t_{0}\right)=\left(\begin{array}{cccc}
0.5 & 0 & 0 & 0 \\
0 & 0.5 & 0 & 0 \\
0 & 0 & 0.1 & 0 \\
0 & 0 & 0 & 0.1
\end{array}\right) .
$$

All motion hypotheses are generated assuming a constant acceleration $\left(a_{k}=a\left(t_{0}\right)\right)$ over the prediction time interval.

The parameters of the prediction time interval are set to $K=25$ and $\Delta t=200 \mathrm{~ms}$ (cf. Eq. (3)), in this way we obtain a maximum prediction time of $t_{25}=4.8 \mathrm{~s}$. If the deviation between the expectation vectors of the object state and the ALP state is below the predetermined distance $\mathcal{B}=$ 0.5 the deviation is set to zero. In this manner we are able to reproduce the behavior of a driver who drives with a constant offset to centerline.

Fig. 6 shows the evaluation of the lane assignment for two vehicles approaching from different sides of the intersection. As one can see in Fig. 6.c the adaptive MCUSUM outperforms the standard MCUSUM, especially if a vehicle has a large offset to the centerline. Compared to the significance level determined by the current Mahalanobis distance, the adaptive MCUSUM also enables more robust decision making. Furthermore, if a lane allows more than one maneuver each of them is covered by a separate motion hypothesis. The significance level of these motion hypotheses are equal to the value of the assigned lane, since no further distinction is possible at this time. Due to the fact that the distance between Obj1 and the centerline 1916 increases from $1.44 \mathrm{~m}$ to $1.6 \mathrm{~m}$ the significance level of this lane decreases simultaneously. At $t=3.1 \mathrm{~s}$ vehicle $\mathrm{Obj} 1$ passes the junction and will be assigned to all successive lanes. For these lanes no history data of the Mahalanobis distances are available so that the significance level starts at 1 . As the vehicle crosses the curve in lane 1522 the orientation of $O b j 1$ and ALP does not match, which leads to a short term reduction of the significance level of this lane. Vehicle $O b j 2$ arrives from lane 1932 to the intersection and departs from it on lane 1534. Since the traffic lanes 1530 and 1534 differ from each other at a late stage, motion hypotheses for both lanes will be generated. At the moment when $O b j 2$ starts to turn right, the significance level of lane 1930 decreases immediately.

Fig. 7 illustrates the influence of the information from the stochastic variable $\mathcal{X}_{A L P}$ on the stochastic estimation process of the EKF. With increasing number of prediction steps the stochastic properties of the estimated object states converge from below to the assumed properties of the ALP, which is shown in Fig. 7b. The convergence rate, however, depends on the variances of the process noise. We performed the motion prediction process with following three covariance matrices of the process noise (cf. Eqn. (21)) to illustrate the effect:

$$
\mathbf{Q}_{1}=\left(\begin{array}{cc}
0.05 & 0 \\
0 & 0.01
\end{array}\right), \mathbf{Q}_{2}=10 \cdot \mathbf{Q}_{1}, \mathbf{Q}_{3}=100 \cdot \mathbf{Q}_{1}
$$

To compare the state-prediction covariance matrix with the covariance matrix of the stochastic variable of the ALP we 


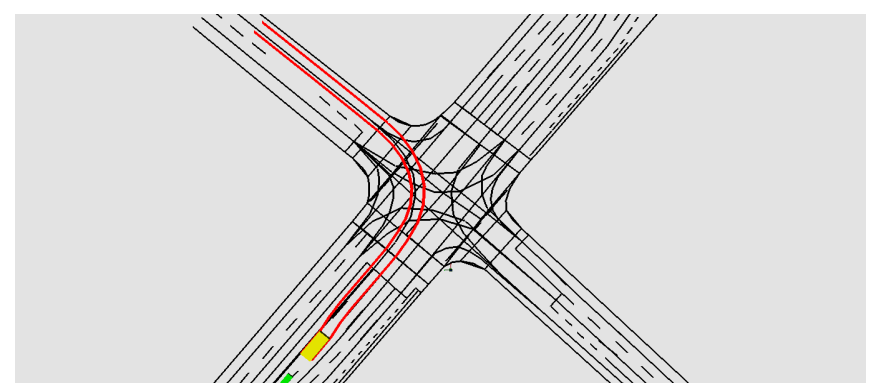

(a) Single motion hypothesis for a vehicle

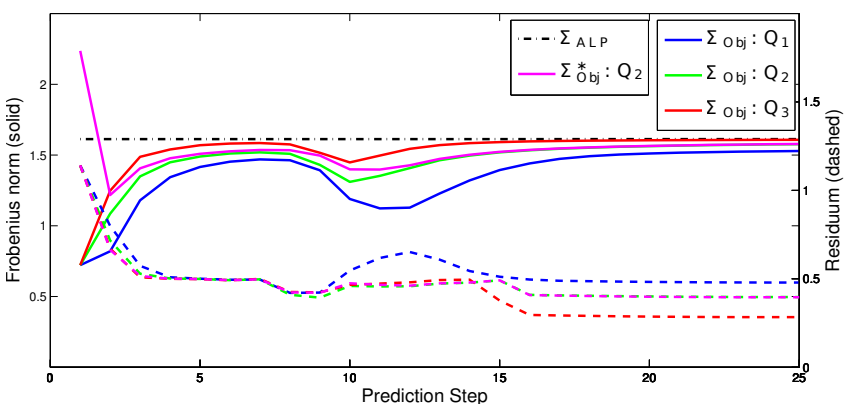

(b) Frobenius norm of the state-prediction covariance matrices and residuum between the predicted expectation vectors of vehicle and ALP

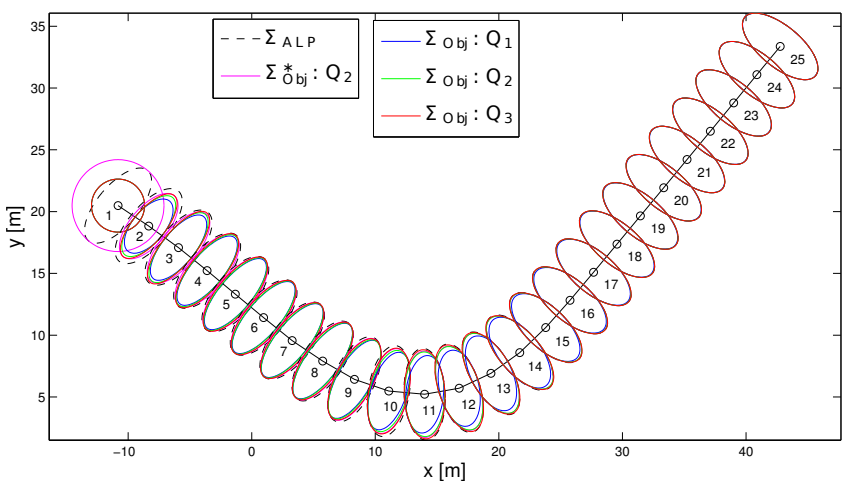

(c) Covariance matrix of the predicted object positions at each prediction step represeted as two-dimensional error ellipses with confidence interval of 0.99 .

Fig. 7. Evolution of the state-prediction covariance matrix for a single motion hypothesis with different Gaussian process noises $\left(\mathbf{Q}_{1}, \mathbf{Q}_{2}\right.$ and $\left.\mathbf{Q}_{3}\right)$. Fig. (a) shows the vehicle on the intersection with one motion prediction, determined by the red edges of the corresponding future motion path. Fig. (b) represents the Frobenius norm of the state-prediction covariance matrices the residuum of the expectation vectors.

employ the Frobenius matrix norm, which is invariant under a unitary transformation. For this reason the Frobenius norm of $\boldsymbol{\Sigma}_{A L P}$ is constant, as shown in Fig. 7.b.

In this manner larger values of process noise lead to an increased convergence rate. This applies to both the covariance matrix and the expectation vector, even if the covariance matrix of the object state is larger than the one of the stochastic representation of the ALP (See Fig. 7 with $\left.\boldsymbol{\Sigma}_{O b j}^{*}=3 \cdot \boldsymbol{\Sigma}_{O b j}\right)$.

\section{CONCLUSIONS}

This paper presents a stochastic filter which is able to generate a representative set of reasonable future trajectories out of the infinite amount of all possible future trajectories for vehicles in traffic environments. This is achieved by incorporating additional information from a digital map into the motion prediction process.

A main benefit of the approach is the assessment of the several motion hypotheses of a particular traffic participants by introducing a new adaptive MCUSUM algorithm. By considering the development over time of the stochastic residual vector between object and ALP state, the adaptive MCUSUM enables more robust decision about whether or not a traffic lane is relevant for a vehicle. Furthermore, it could be demonstrated that with an increasing number of prediction steps, the stochastic properties of the estimated object states converge to the stochastic representation of the centerline information. Convergence rate depends strongly on the modeled process noise.

The presented method predicts the lateral dynamics of detected vehicles. In case of interactions with other traffic participants and surrounding infrastructure we believe that a prediction of transverse dynamics should be possible, too. Our future work will focus on this important issue.

\section{REFERENCES}

[1] A. Tamke, T. Dang, and G. Breuel, "A flexible method for criticality assessment in driver assistance systems," 2011 IEEE Intelligent Vehicles Symposium (IV), no. Iv, pp. 697-702, June 2011.

[2] C. Hermes and C. Wohler, "Long-term vehicle motion prediction," Intelligent Vehicles Symposium, 2009 IEEE, pp. 652-657, 2009.

[3] M. Liebner, F. Klanner, M. Baumann, and C. Ruhhammer, "VelocityBased Driver Intent Inference at Urban Intersections in the Presence of Preceding Vehicles," Intelligent Transportation Systems, 2013, no. April 2013, pp. 10-21, 2013.

[4] E. B. Chun Yang, "Fusion of Tracks with Road Constraints," International Conference on Information Fusion, vol. 3, no. 1, 2008

[5] U. Orguner, T. Schon, and F. Gustafsson, "Improved Target Tracking with Road Network Information,” 2009.

[6] D. Streller, "Road map assisted ground target tracking," International Conference on Fusion, pp. 1162-1168, 2008.

[7] D. Simon and T. Chia, "Kalman filtering with state equality constraints," Aerospace and Electronic Systems, IEEE, vol. 38, no. 1, 2002

[8] C. Hasberg, S. Hensel, M. Westenkirchner, and K. Bach, "Integrating Spline Curves in Road Constraint Object Tracking," Intelligent Transportation Systems, 2008, pp. 1009-1014, 2008.

[9] M. Ulmke and W. Koch, "Road-map assisted ground moving target tracking," Aerospace and Electronic Systems, IEEE, vol. 42, no. 4, 2006.

[10] C. Yang, M. Bakich, and E. Blasch, "Nonlinear constrained tracking of targets on roads," Information Fusion, 2005 8th International Conference on. IEEE, 2005.

[11] "Ko-FAS - Ko-PER - Cooperative Perception." [Online]. Available: http://ko-fas.de/english/ko-per-cooperative-perception.html

[12] G. Welch and G. Bishop, "An introduction to the Kalman filter," pp. $1-16,1995$

[13] "World Geodetic System 1984, Its Definition and Relationships with Local Geodetic Systems," Tech. Rep.

[14] J. Yong and F. Cheng, "Geometric Hermite curves with minimum strain energy," Computer Aided Geometric Design, vol. 21, pp. 281301, 2004.

[15] M. Mitschke and H. Wallentowitz, "Dynamik der Kraftfahrzeuge," 2004.

[16] Y. Dai, Y. Luo, Z. Li, and Z. Wang, "A new adaptive CUSUM control chart for detecting the multivariate process mean," Quality and Reliability Engineering International, pp. 877-884, 2011. 